\title{
Tunneling through ultrasmall NIS junctions in terms of Andreev reflection: a nonlinear response approach
}

\author{
A. Hädicke and W. Krech \\ Institut für Festkörperphysik, Friedrich-Schiller-Universität Jena, Max-Wien-Platz 1 \\ D-07743 Jena, Germany
}

(April 1995)

\begin{abstract}
The Andreev current through an ultrasmall NIS junction is calculated in a systematic way by means of a nonlinear response approach basing on the elementary Hamiltonian of quasiparticle tunneling. The voltage dependence of current and differential conductance as well as the Andreev conductance are derived for low- and high-impedance environments, respectively.
\end{abstract}

$73.40,74.50$

\section{INTRODUCTION}

Effects of single-charge tunneling at junctions with ultrasmall capacitances have been studied both theoretically and experimentally during the last years. For a review see [1]. Modern nanolithography allows the fabrication of tunnel junctions with capacitances less than $C<10^{-16} \mathrm{~F}$ which means that thermal fluctuations can be disregarded at the $1 \mathrm{~K}$ scale. Since the tunnel resistances $R$ satisfy the condition $R \gg R_{Q}$ quantum fluctuations can be neglected. $R_{Q}$ is the quantum resistance $R_{Q}=h / e^{2}$. Different types of junctions and several charge transport mechanisms depending on whether the electrodes are normal or superconducting ones have been investigated.

Here the simple example of a voltage driven NIS junction in series with an environment resistance $R_{E}$ is studied at zero temperature. At $T=0$ there is no charge transport through a NIS junction in terms of quasiparticles for voltages lower than the threshold $\Delta / e$, where $2 \Delta$ is the energy gap of the superconductor $(\Delta \equiv \Delta(0))$. The reason is that there are no quasiparticles in the superconductor with energies below $\Delta$. The quasiparticle current $\langle I\rangle_{q p}$ reads [2]

$$
\langle I\rangle_{q p}(V)=\frac{1}{R} \sqrt{V^{2}-\left(\frac{\Delta}{e}\right)^{2}} \Theta\left(V-\frac{\Delta}{e}\right)-[V \rightarrow-V],
$$

where the symbol $\Theta$ stands for the unit step function. Eq. (1) is valid at $T=0$ and in case of a vanishing environment resistance $\left(R_{E} / R_{Q} \rightarrow 0\right)$. Since the electromagnetic environment is able to absorb energy it influences the currentvoltage characteristic of a single junction essentially. In the limit of a high-resistance environment $\left(R_{E} / R_{Q} \rightarrow \infty\right)$ the voltage threshold $\Delta / e$ is increased by the Coulomb blockade $E_{c} / e=e /(2 C)$. The corresponding expression of the current can be get from Eq. 11 by the replacement $V \rightarrow V-E_{c} / e$;

$$
\langle I\rangle_{q p}(V)=\frac{1}{R} \sqrt{\left(V-\frac{E_{c}}{e}\right)^{2}-\left(\frac{\Delta}{e}\right)^{2}} \Theta\left(V-\frac{E_{c}}{e}-\frac{\Delta}{e}\right)-[V \rightarrow-V] .
$$

But at the moment let us ignore the environment. There is another charge transport mechanism leading to a nonvanishing current in the the subgap region $(0<V<\Delta / e)$. It corresponds to the transfer of two electrons which can be converted into a Cooperpair instantaneously. This process, known as Andreev reflection in case of no barrier for a long time [3], exists under subgap conditions because it avoids the generation of excited states in the superconductor. In this connection the influence of imperfections in the barrier or the electrodes as well as the proximity effect is disregarded. The current which is much smaller than ordinary quasiparticle tunneling (Eq. 1) corresponds to a process of higher order in perturbation theory and the conductivity emerges to be proportional to $R^{-2}$. The effect was described by means of the Bogoliubov-de Gennes equations [- $[$ as well as in terms of a transition rate formulation [5] 9]. Here, we are going to show how the nonlinear response approach basing on the elementary Hamiltonian of quasiparticle tunneling generates in a systematic and direct way those current contributions (beside others) which correspond to the Andreev reflection. After sketching the model (Sec. II) and basic concepts of nonlinear response theory (Sec. III), we derive the Andreev current (Secs. IV and V) for the low-impedance and the high-impedance cases (Sec. VII), respectively. We restrict ourselves to the concept of ballistic motion of electrons. Finally, a simple approximation in case of the high-impedance environment is given (Sec. VIII). 


\section{THE MODEL}

The Hamiltonian of a voltage driven NIS junction with environment reads

$$
H=Q V+H_{\text {res }}+H_{T},
$$

where the tunnel Hamiltonian is given by

$$
\begin{array}{ll}
H_{T} & =H_{+}+H_{-}, \\
H_{+} & =\sum_{l, r, \sigma} T_{l r} c_{r, \sigma}^{\dagger} c_{l, \sigma} e^{i \Phi} .
\end{array}
$$

$c_{l, \sigma}$ and $c_{r, \sigma}$ stand for electron annihilation operators of the left and right electrode, respectively, satisfying anticommutation relations. The spin is labeled by the subscript $\sigma$. The sum is taken over momenta $l$ and $r$ and the spin $(\sigma=\uparrow, \downarrow)$. The reservoir Hamiltonian $H_{\text {res }}=H_{L}+H_{R}+H_{E}$ consists of terms corresponding to the left $(L)$ and right $(R)$ electrodes and the environment $(E)$ which can be described in standard way [10,11]. Owing to the phase operator tunneling is connected with excitations in the electromagnetic environment. The convention is chosen in such a way that a positive voltage favors tunneling from left to right which reduces the junction charge $Q$ by $e$. The operator $H_{+}$ means tunneling from left to right in contrast to the Hermitian conjugate which describes the reverse process. $T_{l r}$ are the tunneling matrix elements. The basic algebra ruling this approach is the relation 12

$$
H_{ \pm} \cdot F(Q)=F(Q \pm e) \cdot H_{ \pm}
$$

where $F$ is an arbitrary function of the junction charge $Q$. The operator $\exp ( \pm i \Phi)$ changes the macroscopic charge $Q$ on the junction by the value $\mp e$. The algebra (5) corresponds to the elementary commutation relation

$$
[Q, \Phi]=i e .
$$

An important point is to take into account the specific feature of a NIS junction. Without loss of generality we assume that the right electrode is the superconducting one. Therefore, Bogoliubov transformed operators are introduced on the right-hand side in the following way

$$
\begin{aligned}
& c_{r, \sigma}=u_{r, \sigma} \gamma_{r, \sigma}+v_{r, \sigma} \gamma_{-r,-\sigma}^{\dagger}, \\
& c_{r, \sigma}^{\dagger}=u_{r, \sigma} \gamma_{r, \sigma}^{\dagger}+v_{r, \sigma} \gamma_{-r,-\sigma},
\end{aligned}
$$

where $\gamma_{r, \sigma}$ and $\gamma_{r, \sigma}^{\dagger}$ are new Fermi operators satisfying common anticommutation relations and the numerical coefficients $u_{r, \sigma}$ and $v_{r, \sigma}$ are the known BCS coherence factors [2]. We remind of the off-diagonal Hamiltonian of the superconducting electrode which becomes diagonal in the new operators $\gamma^{\dagger}$ and $\gamma$

$$
H_{R}=\mathrm{const}+\sum_{r, \sigma} E_{r} \gamma_{r, \sigma}^{\dagger} \gamma_{r, \sigma} .
$$

The quasiparticle energies of the superconductor read

$$
E_{r}=\sqrt{\varepsilon_{r}^{2}+\Delta^{2}} .
$$

$H_{L}$ on the left-hand side is given by

$$
H_{L}=\sum_{l, \sigma} \varepsilon_{l} c_{l, \sigma}^{\dagger} c_{l, \sigma},
$$

where $\varepsilon_{l}$ are the usual electron energies counted with respect to the Fermi energy.

\section{NONLINEAR RESPONSE THEORY}

The dynamics of our physical system modeled by an unperturbed Hamiltonian $H_{o}$ and an interaction (tunneling) $H_{T}$ is described by the statistical operator $\rho$ satisfying the von Neumann equation which corresponds in the interaction representation (superscript $(I)$ ) to the integral equation 


$$
\rho(t)^{(I)}=\rho_{o}-\frac{i}{\hbar} \int_{-\infty}^{t}\left[H_{T}^{(I)}\left(t^{\prime}\right), \rho^{(I)}\left(t^{\prime}\right)\right] \mathrm{d} t^{\prime} .
$$

It is assumed that the interaction is switched on at $t=-\infty$ adiabatically. $\rho_{o}$ is given by the canonical expression $\left(\beta=1 /\left(k_{B} T\right)\right)$

$$
\rho_{o}=\frac{e^{-\beta H_{o}}}{\operatorname{tr}\left\{e^{-\beta H_{o}}\right\}} .
$$

The mean value of an arbitrary operator can be calculated in terms of a successive approximation. In case of the current operator defined by

$$
I=-\frac{\mathrm{d}}{\mathrm{d} t} Q=-\frac{1}{i \hbar}[Q, H]=\frac{e}{i \hbar}\left(H_{+}-H_{-}\right)
$$

one finds the expansion

$$
\begin{aligned}
\langle I\rangle= & \frac{1}{i \hbar} \int_{-\infty}^{t} \mathrm{~d} t^{\prime}\left\langle\left[I^{(I)}(t), H_{T}^{(I)}\left(t^{\prime}\right)\right]\right\rangle_{o} \\
& +\left(\frac{1}{i \hbar}\right)^{3} \int_{-\infty}^{t} \mathrm{~d} t^{\prime} \int_{-\infty}^{t^{\prime}} \mathrm{d} t^{\prime} \int_{-\infty}^{t^{\prime \prime}} \mathrm{d} t^{\prime \prime \prime}\left\langle\left[\left[\left[I^{(I)}(t), H_{T}^{(I)}\left(t^{\prime}\right)\right], H_{T}^{(I)}\left(t^{\prime \prime}\right)\right], H_{T}^{(I)}\left(t^{\prime \prime \prime}\right)\right]\right\rangle_{o}+\ldots
\end{aligned}
$$

A term of zeroth order is missing because in case of no interaction (tunneling) there is also no current. Now the quasiparticle current according to the first order term in Eq. (12) can be calculated. The result

$$
\langle I\rangle_{q p}=-\frac{2 e}{\hbar^{2}} \operatorname{Re} \int_{-\infty}^{t} \mathrm{~d} t^{\prime}\left\langle\left[H_{+}^{(I)}(t), H_{-}^{(I)}\left(t^{\prime}\right)\right]\right\rangle_{o}
$$

leads at $T=0$ to the Eqs. (11) and (2), respectively. The Andreev current is contained in the following terms of second nonvanishing order

$$
\begin{aligned}
\langle I\rangle=\frac{2 e}{\hbar^{4}} \operatorname{Re} \int_{-\infty}^{t} \mathrm{~d} t^{\prime} \int_{-\infty}^{t^{\prime}} \mathrm{d} t^{\prime \prime} \int_{-\infty}^{t^{\prime \prime}} \mathrm{d} t^{\prime \prime \prime}\{ & \left\langle\left[\left[\left[H_{+}^{(I)}(t), H_{+}^{(I)}\left(t^{\prime}\right)\right], H_{-}^{(I)}\left(t^{\prime \prime}\right)\right], H_{-}^{(I)}\left(t^{\prime \prime \prime}\right)\right]\right\rangle_{o} \\
+ & {\left.\left[\left[\left[H_{+}^{(I)}(t), H_{-}^{(I)}\left(t^{\prime}\right)\right], H_{+}^{(I)}\left(t^{\prime \prime}\right)\right], H_{-}^{(I)}\left(t^{\prime \prime \prime}\right)\right]\right\rangle_{o} } \\
+ & {\left.\left.\left[\left[\left[H_{+}^{(I)}(t), H_{-}^{(I)}\left(t^{\prime}\right)\right], H_{-}^{(I)}\left(t^{\prime \prime}\right)\right], H_{+}^{(I)}\left(t^{\prime \prime \prime}\right)\right]\right\rangle_{o}\right\} }
\end{aligned}
$$

where the angle brackets $\langle\ldots\rangle_{o}$ denote averaging with respect to $\rho_{o}$. In Eq. (14) only correlations with vanishing signature $(++--$ and their permutations) are taken into account. The reason is that the separation of the voltage dependence in the correlation functions $\left(\langle\ldots\rangle_{o}\right)$ with nonvanishing signature (e.g. $\left.\left\langle H_{+} H_{+} H_{+} H_{+}\right\rangle_{o}\right)$ leads to expressions containing time dependent $(t)$ terms. Furthermore, a phase $\Phi_{o}$ remains indeterminated additionally. This is like in common Josephson physics where contributions proportional to $\sin \left(2 e V t / \hbar+\Phi_{o}\right)$ and $\cos \left(2 e V t / \hbar+\Phi_{o}\right)[13$ arise. However, with respect to the Josephson effect the phase $\Phi_{o}$ is adjusted by current biasing. Therefore, in our opposite case of voltage biasing, where the charge becomes adjusted, these terms do not contribute.

According to the fact that Cooper pairs live in the condensate the corresponding energy balance of the Andreev current does not contain any energy contributions belonging to the superconducting bank. It turns out that the Andreev current is only determined by the first summand of Eq. (14) because the energy balances of the two other summands always depend on the quasiparticle energies $E_{r}$. 


\section{CALCULATION OF THE CORRELATION FUNCTION}

The voltage dependence can be separated by means of Eq. (5) and in terms of the new time variables

$$
\tau \equiv t-t^{\prime} ; \quad \tau^{\prime} \equiv t^{\prime}-t^{\prime \prime} ; \quad \tau^{\prime \prime} \equiv t^{\prime \prime}-t^{\prime \prime \prime}
$$

one gets the Andreev current $\langle I\rangle^{(A)}$

$$
\langle I\rangle^{(A)}=\frac{2 e}{\hbar^{4}} \operatorname{Re} \int_{0}^{\infty} \mathrm{d} \tau \int_{0}^{\infty} \mathrm{d} \tau^{\prime} \int_{0}^{\infty} \mathrm{d} \tau^{\prime \prime} e^{-\frac{i}{\hbar} e V\left(\tau+2 \tau^{\prime}+\tau^{\prime \prime}\right)} \kappa\left(\tau, \tau^{\prime}, \tau^{\prime \prime}\right)
$$

with

$$
\kappa\left(\tau, \tau^{\prime}, \tau^{\prime \prime}\right)=\left\langle\left[\left[\left[\hat{H}_{+}^{(I)}(t), \hat{H}_{+}^{(I)}(t-\tau)\right], \hat{H}_{-}^{(I)}\left(t-\tau-\tau^{\prime}\right)\right], \hat{H}_{-}^{(I)}\left(t-\tau-\tau^{\prime}-\tau^{\prime \prime}\right)\right]\right\rangle_{o} .
$$

The hat indicates that the operators only carry the time dependence with respect to the electrodes and the environment. The resolution of the interlaced commutators and the separation of the environment lead to

$$
\begin{aligned}
\kappa= & -\left\langle\tilde{H}_{-}\left(t-\tau-\tau^{\prime}-\tau^{\prime \prime}\right) \tilde{H}_{-}\left(t-\tau-\tau^{\prime}\right) \tilde{H}_{+}(t-\tau) \tilde{H}_{+}(t)\right\rangle_{o} \\
& \times\left\langle e^{-i \Phi\left(t-\tau-\tau^{\prime}-\tau^{\prime \prime}\right)} e^{-i \Phi\left(t-\tau-\tau^{\prime}\right)} e^{i \Phi(t-\tau)} e^{i \Phi(t)}\right\rangle_{o}+7 \text { further terms } .
\end{aligned}
$$

Now the operators $\tilde{H}_{ \pm}$only possess the time dependence with respect to the electrodes

$$
\tilde{H}_{ \pm}(t)=e^{\frac{i}{\hbar}\left(H_{L}+H_{R}\right) t} H_{ \pm} e^{-\frac{i}{\hbar}\left(H_{L}+H_{R}\right) t} .
$$

To simplify matters at first we restrict ourselves to a low-resistance environment $\left(R_{E} \ll R_{Q}\right)$. This means that the phase correlation functions can be replaced by 1 . The high-resistance case is dealt with in Sec. VI. The calculation of the correlation function $\kappa$ is rather lengthy and cannot be shown in detail. The operators $H_{ \pm}$have to be expressed by the elementary operators $c, c^{\dagger}, \gamma$ and $\gamma^{\dagger}$ which show an exponential time dependence:

$$
\begin{aligned}
c_{l, \sigma}^{(\dagger)}(t) & =e^{ \pm \frac{i}{\hbar} \varepsilon_{l} \cdot t} c_{l, \sigma}^{(\dagger)}, \\
\gamma_{r, \sigma}^{(\dagger)}(t) & =e^{ \pm \frac{i}{\hbar} E_{r} \cdot t} \gamma_{r, \sigma}^{(\dagger)} .
\end{aligned}
$$

Using Wick's theorem, the correlations can be expressed by the Fermi distribution functions $f$ and the different time integrations can be carried out. By means of Dirac's formula, the real part can be taken and the relevant contribution coming from the first term in Eq. (17) turns out to be

$$
\begin{aligned}
\frac{8 e \pi}{\hbar} \sum_{\substack{l l^{\prime} \\
r r^{\prime}}} & f\left(\varepsilon_{l}\right) f\left(\varepsilon_{l^{\prime}}\right) f\left(-E_{r}\right) f\left(-E_{r^{\prime}}\right) u_{r} v_{r} u_{r^{\prime}} v_{r^{\prime}} 2 T_{l r}^{*} T_{l^{\prime}-r}^{*} T_{l r^{\prime}} T_{l^{\prime}-r^{\prime}} \delta\left(-\varepsilon_{l}-\varepsilon_{l^{\prime}}-2 e V\right) \\
\times & {\left[\frac{1}{E_{r}-\varepsilon_{l}-e V} \frac{1}{E_{r^{\prime}}-\varepsilon_{l^{\prime}}-e V}+\frac{1}{E_{r}-\varepsilon_{l}-e V} \frac{1}{E_{r^{\prime}}-\varepsilon_{l}-e V}\right] . }
\end{aligned}
$$

The summation over the spin indices has already been performed. The derivation of Eq. (20) uses the properties

$$
u_{r, \sigma}=u_{r,-\sigma} ; \quad v_{r, \sigma}=-v_{r,-\sigma} .
$$

During the calculation one has to treat lots of terms but only a few of them are nonvanishing and contribute to the Andreev current. In similar way as above this analysis can be applied to the other seven terms in Eq. (17).

\section{ANDREEV CURRENT}

Further calculations require an assumption of the momentum dependence of the tunneling matrix elements. With respect to quasiparticle tunneling the squared absolute values of tunnel matrix elements $\left|T_{l r}\right|^{2}$ emerge which are approximated by their momentum averages at the Fermi edges of the electrodes $\left\langle\left|T_{l r}\right|^{2}\right\rangle$ usually. Furthermore, the phenomenological tunnel conductance is defined by 


$$
G \equiv \frac{2 \pi e^{2}}{\hbar} \nu(0)^{2} 2\left\langle\left|T_{l r}\right|^{2}\right\rangle,
$$

where $\nu(0)$ is the density of states at the Fermi level in the normal conducting state. In contrast to this the product of tunnel matrix elements in Eq. (20) is much more complicated because it depends crucially on the nature of the electron motion. Some effort has been done to treat this in terms of a diffusive transport [14,7]. However, in case of sufficiently small junctions we can assume that the picture of ballistic electron motion is correct which means that the scattering of electrons can be neglected. We follow Ref. [5] and approximate the momentum averaged product of tunneling matrix elements $2\left\langle T_{l r}^{*} T_{l^{\prime}-r}^{*} T_{l r^{\prime}} T_{l^{\prime}-r^{\prime}}\right\rangle$ in terms of $2\left\langle\left|T_{l r}\right|^{2}\right\rangle \cdot 2\left\langle\left|T_{l^{\prime} r^{\prime}}\right|^{2}\right\rangle / N$, where $N$ is a correction factor which depends on the geometry of the junction. It corresponds to the number of independent current channels. The additional factors 2 are due to the spin. Now, the discrete sums are transformed into integrations where the energy densities of states of the superconductor and the normal conductor arise. Finally, the Andreev current is given by the following expression:

$$
\begin{aligned}
\langle I\rangle^{(A)}(V)= & \frac{\hbar G^{2} \Delta^{2}}{\pi e^{3} N} \int_{-\infty}^{\infty} \mathrm{d} \varepsilon_{l} \int_{-\infty}^{\infty} \mathrm{d} \varepsilon_{l^{\prime}} \int_{-\infty}^{\infty} \mathrm{d} E_{r} \int_{-\infty}^{\infty} \mathrm{d} E_{r^{\prime}} f\left(\varepsilon_{l}\right) f\left(\varepsilon_{l^{\prime}}\right) f\left(-E_{r}\right) f\left(-E_{r^{\prime}}\right) \frac{\Theta\left(\left|E_{r}\right|-\Delta\right)}{\sqrt{E_{r}^{2}-\Delta^{2}}} \frac{\Theta\left(\left|E_{r^{\prime}}\right|-\Delta\right)}{\sqrt{E_{r^{\prime}}^{2}-\Delta^{2}}} \\
& \times\left[\frac{1}{E_{r}-\varepsilon_{l}-e V} \frac{1}{E_{r^{\prime}}-\varepsilon_{l^{\prime}}-e V}+\frac{1}{E_{r}-\varepsilon_{l}-e V} \frac{1}{E_{r^{\prime}}-\varepsilon_{l}-e V}\right] \delta\left(-\varepsilon_{l}-\varepsilon_{l^{\prime}}-2 e V\right) .
\end{aligned}
$$

At zero temperature the Fermi distribution becomes a theta function $(f(x)=\Theta(-x))$ and the energy integrations can be carried out. The resulting formula of the Andreev current

$$
\langle I\rangle^{(A)}(V)=\frac{\pi}{2} \frac{\hbar G^{2} \Delta}{e^{3} N} \log \left(\frac{1+\frac{e V}{\Delta}}{1-\frac{e V}{\Delta}}\right) \quad \text { for } \quad-\frac{\Delta}{e}<V<\frac{\Delta}{e}
$$

shows logarithmic singularities at $V= \pm \Delta / e$. This is not surprising because $\Delta / e$ corresponds just to the voltage threshold of quasiparticle tunneling and a singularity of such kind is known from inelastic co-tunneling [15]. The singularity which is an artefact of perturbation theory shows that still higher order terms are necessary to describe the crossover between quasiparticle and Andreev tunneling. The divergence disappears leaving a finite enhancement if a finite lifetime broadening is taken into account. This can be done either by hand [16] or by performing a nonperturbative resummation technique [17,18]. Of course, the singularity will also be smoothed both due to finite temperatures and finite environment resistances. A Taylor expansion in terms of the voltage $(V \ll \Delta / e)$ yields the linear behavior

$$
\langle I\rangle^{(A)}(V) \approx \pi \frac{\hbar G^{2}}{e^{2} N} V
$$

and the Andreev conductance

$$
G^{(A)}=\left.\frac{\mathrm{d}\langle I\rangle^{(A)}(V)}{\mathrm{d} V}\right|_{V=0}=\frac{\pi \hbar G^{2}}{e^{2} N}=\frac{R_{Q} G^{2}}{2 N} .
$$

Note, that $G^{(A)}$ is proportional to $R^{-2}$. Fig. 1 shows the Andreev current (23) and its linear approximation (24) in the subgap region of quasiparticle tunneling. The scale of quasiparticle tunneling beyond the threshold is about several orders of magnitude (factor $2 N /\left(R_{Q} G\right) \gg 1$ ) larger than that of the Andreev tunneling.

\section{HIGH-IMPEDANCE CASE}

The investigation of the high-impedance case is based on Eq. (17). Now we have to calculate the phase correlation functions which were so far replaced by 1 . This can be done in Gaussian approximation, for instance by generalizing the method presented in Ref. [19]. The phase correlation functions occuring in Eq. (17) depend on the function $J$ which contains the information about the structure of the environment. See e. g. Refs. [10,11, 19]. In the high-impedance case and at $T=0$ there is a linear behavior

$$
J(\tau)=-i \frac{E_{c}}{\hbar} \tau
$$


which leads to the following expression for the phase correlation function of first term of Eq. (17)

$$
\left\langle e^{-i \Phi\left(t-\tau-\tau^{\prime}-\tau^{\prime \prime}\right)} e^{-i \Phi\left(t-\tau-\tau^{\prime}\right)} e^{i \Phi(t-\tau)} e^{i \Phi(t)}\right\rangle_{o}=e^{i \frac{E_{c}}{\hbar}\left(\tau+4 \tau^{\prime}+\tau^{\prime \prime}\right)} .
$$

Now the first term of Eq. (17) reads

$$
\begin{aligned}
\frac{8 e \pi}{\hbar} \sum_{\substack{l l^{\prime} \\
r r^{\prime}}} & f\left(\varepsilon_{l}\right) f\left(\varepsilon_{l^{\prime}}\right) f\left(-E_{r}\right) f\left(-E_{r^{\prime}}\right) u_{r} v_{r} u_{r^{\prime}} v_{r^{\prime}} 2 T_{l r}^{*} T_{l^{\prime}-r}^{*} T_{l r^{\prime}} T_{l^{\prime}-r^{\prime}} \delta\left(-\varepsilon_{l}-\varepsilon_{l^{\prime}}-2 e V+4 E_{c}\right) \\
\times & {\left[\frac{1}{E_{r}-\varepsilon_{l}-e V+E_{c}} \frac{1}{E_{r^{\prime}}-\varepsilon_{l^{\prime}}-e V+E_{c}}+\frac{1}{E_{r}-\varepsilon_{l}-e V+E_{c}} \frac{1}{E_{r^{\prime}}-\varepsilon_{l}-e V+E_{c}}\right] . }
\end{aligned}
$$

Finally, taking all relevant contributions into account, one gets the Andreev current

$$
\langle I\rangle^{(A)}(V)=\frac{\hbar G^{2} \Delta}{e^{3} N} \frac{1}{2 \pi} \int_{-\hat{v}}^{\hat{v}} \mathrm{~d} z\left[\int_{1}^{\infty} \mathrm{d} y \frac{1}{\sqrt{y^{2}-1}} \frac{2(y-w)}{(y-w)^{2}-z^{2}}\right]^{2} \Theta\left(e V-2 E_{c}\right)-[V \rightarrow-V],
$$

where the abbreviations $w=E_{c} / \Delta$ and $\hat{v}=e V / \Delta-2 w$ are used (see Fig. 2, solid line). Eq. (29) is valid in the interval $-\left(\Delta+E_{c}\right) / e<V<\left(\Delta+E_{c}\right) / e$. Of course, the Coulomb blockade of Andreev tunneling $\left(V_{b l}^{(A)}=2 E_{c} / e\right)$ should be smaller than the threshold of quasiparticle tunneling $\left(\left(\Delta+E_{c}\right) / e\right)$. This is guaranteed for $E_{c}<\Delta$. Though the Andreev current cannot be calculated analytically we are able to derive the differential conductance $g(V)$ (see Fig. 3, solid line)

$$
g(V)=\frac{\mathrm{d}\langle I\rangle^{(A)}(V)}{\mathrm{d} V}=\frac{R_{Q} G^{2}}{2 N} \frac{1}{\pi^{2}}\left[\frac{\frac{\pi}{2}+\arcsin (w-\hat{v})}{\sqrt{1-(w-\hat{v})^{2}}}+\frac{\frac{\pi}{2}+\arcsin (w+\hat{v})}{\sqrt{1-(w+\hat{v})^{2}}}\right]^{2} \Theta\left(e V-2 E_{c}\right)+[V \rightarrow-V] .
$$

Hence, the Andreev conductance which is defined as the differential conductance at the blockade voltage $V_{b l}^{(A)}$ turns out to be

$$
G^{(A)}=g\left(V_{b l}^{(A)}\right)=\frac{R_{Q} G^{2}}{2 N} \frac{\left(1+\frac{2}{\pi} \arcsin w\right)^{2}}{1-w^{2}}=\frac{R_{Q} G^{2}}{2 N} \frac{\left(\frac{4}{\pi} \arctan \frac{\sqrt{1+w}}{\sqrt{1-w}}\right)^{2}}{1-w^{2}} .
$$

The result coincides with that of Ref. [5]. Eq. 25) is reproduced in the limit $E_{c} \rightarrow 0$. Fig. 1 (solid line) shows the Andreev conductance as a function of $w=E_{c} / \Delta$. In case that the Coulomb blockade $V_{b l}^{(A)}=2 E_{c} / e$ tends to the voltage threshold of quasiparticle tunneling $\left(\Delta+E_{c}\right) / e$ the Andreev conductance becomes singular $(w \rightarrow 1)$.

\section{APPROXIMATION OF ANDREEV CURRENT}

Since the Andreev current (cf. Eq. (29)) is given by an integral which cannot be carried out analytically, we want to derive an approximated expression. This can be achieved by taking into account only the relevant singularities of the integrand, namely $1 /\left(1-(w-z)^{2}\right)$ and $1 /\left(1-(w+z)^{2}\right)$. According to that, the expresion in squared brackets in Eq. (29) is approximated in the following way:

$$
[\ldots]^{2} \approx \frac{a_{1}}{1-(w-z)^{2}}+\frac{a_{2}}{1-(w+z)^{2}},
$$

where $a_{1}$ and $a_{2}$ are constants. Now the integration can be carried out and we get

$$
\frac{\hbar G^{2} \Delta}{e^{3} N} \frac{a^{*}}{4 \pi} \log \left(\frac{1+w+\hat{v}}{1+w-\hat{v}} \cdot \frac{1-w+\hat{v}}{1-w-\hat{v}}\right) \Theta(\hat{v})-[V \rightarrow-V],
$$

where a new constant $a^{*}=a_{1}+a_{2}$ is introduced. Furthermore, the factor $(1+w+\hat{v}) /(1+w-\hat{v})$ in the argument of the logarithm can be neglected because it is an irrelevant contribution. The unknown constant $a^{*}$ is determined by the demand 


$$
\left.G_{\text {approx }}^{(A)}\right|_{w=0}=\left.\frac{\hbar G^{2}}{e^{2} N} \frac{a^{*}}{2 \pi} \equiv G^{(A)}\right|_{w=0}=\frac{R_{Q} G^{2}}{2 N},
$$

which gives $a^{*}=2 \pi^{2}$. Hence, the approximated Andreev current reads

$$
\langle I\rangle_{\text {approx }}^{(A)}(V) \approx \frac{R_{Q} G^{2}}{2 N} \frac{\Delta}{2 e} \log \frac{\Delta-3 E_{c}+e V}{\Delta+E_{c}-e V} \Theta\left(e V-2 E_{c}\right)-[V \rightarrow-V] .
$$

We have plotted both the exact and the approximated Andreev current as functions of the voltage for $2 E_{c}<\mathrm{eV}<$ $\Delta+E_{c}$ in Fig. 2. As an example $E_{c}$ is chosen to be $E_{c}=\Delta / 2$. The approximated differential conductance is given by differentiation

$$
g(V)=\frac{R_{Q} G^{2}}{2 N} \frac{1-w}{(1-w+\hat{v})(1-w-\hat{v})} \Theta\left(e V-2 E_{c}\right)+[V \rightarrow-V]
$$

and can be compared with the exact expression (30) (see Fig. 3). The approximated Andreev conductance reads

$$
G_{\mathrm{approx}}^{(A)}=\frac{R_{Q} G^{2}}{2 N} \frac{1}{1-w}
$$

Fig. 4 shows both the exact and the approximated expressions of the Andreev conductance as a function of $w=E_{c} / \Delta$.

\section{ACKNOWLEDGMENTS}

We would like to thank H.-O. Mller for helpful discussions. This work was supported by the Deutsche Forschungsgemeinschaft.

\section{CONCLUSION}

In conclusion we have shown how the Andreev current through a NIS junction can be calculated by means of a nonlinear response approach basing on the elementary Hamiltonian of quasiparticle tunneling. This method generates in a straightforward way all kinds of current contributions including quasiparticle and Andreev tunneling. For low- and high-resistance environments we have calculated the voltage dependence of the Andreev current and the differential conductance explicitly. Finally, a simple approximation in case of the high-impedance environment has been given.

[1] H. Grabert and M. H. Devoret, Single Charge Tunneling: Coulomb Blockade Phenomena in Nanostructures, NATO ASI Series B: Physics, Vol. 294, (Plenum, New York and London, 1992).

[2] M. Tinkham, Introduction to Superconductivity, (McGraw-Hill, New York, 1976).

[3] A. F. Andreev, Zh. Eksp. Teor. Fiz. 46, 1823 (1964).

[4] G. E. Blonder, M. Tinkam and T. M. Klapwijk, Phys. Rev. B 25, 4515 (1982).

[5] F. W. J. Hekking, L. I. Glazman, K. A. Matveev and R. I. Shekhter, Phys. Rev. Lett. 70, 4138 (1993).

[6] F. W. J. Hekking and Yu. V. Nazarov, Phys. Rev. Lett. 71, 1625 (1993).

[7] F. W. J. Hekking and Yu. V. Nazarov, Phys. Rev. B 49, 6847 (1994).

[8] J. J. Hesse and G. Diener, Physica B 203, 393 (1994).

[9] U. Hanke, ShotNoise Spectra in Single Electron Tunnel Junctions, (PhD thesis, University of Trondheim 1994).

[10] M. H. Devoret, D. Esteve, H. Grabert, G.-L. Ingold, H. Pothier and C. Urbina, Phys. Rev. Lett. 64, 1824 (1990).

[11] H. Grabert, G.-L. Ingold, M. H. Devoret, D. Esteve, H. Pothier and C. Urbina, Z. Phys. B 84, 143 (1991).

[12] D. V. Averin and K. K. Likharev, J. Low Temp. Phys. 62, 345 (1986).

[13] D. Rogovin and D. J. Scalapino, Ann. Phys. (N.Y.) 86, 1 (1974).

[14] D. V. Averin and Yu. V. Nazarov, Phys. Rev. Lett. 65, 2446 (1990); in Ref. [1], pp. 217-247.

[15] D. V. Averin and A. A. Odintsov, Phys. Lett. A 140251 (1989).

[16] W. Krech and A. Hädicke, Int. J. Mod. Phys. B 7, 2201 (1992). 
[17] P. Lafarge and D. Esteve, Phys. Rev. B 48, 14309 (1993).

[18] H. Schoeller and G. Schön, Mesoscopic quantum transport: Resonant tunneling in the presence of strong Coulomb interaction, (preprint 1994).

[19] G.-L. Ingold and Yu. V. Nazarov, in Ref. 迎, pp. 21-107.

FIG. 1. The Andreev current in the low-impedance case (Eq. (23), solid line) and its linear expansion (Eq. (24), dashed line) are plotted versus the voltage in the interval $0<e V / \Delta$. Current and voltage are counted in units of $R_{Q} G^{2} \Delta /(2 N e)$ and $\Delta / e$, respectively.

FIG. 2. The Andreev current in the high-impedance case (Eq. (29), solid line) and its approximation (Eq. 34), dashed line) are plotted versus the voltage in the interval $2 E_{c}<e V<\Delta+E_{c}$ for $E_{c}=\Delta / 2$. Current and voltage are counted in units of $R_{Q} G^{2} \Delta /(2 N e)$ and $\Delta / e$, respectively.

FIG. 3. The differential conductance (Eq. (30), solid line) and its approximation (Eq. (35), dashed line) are drawn as functions of the voltage for $2 E_{c}<e V<\Delta+E_{c}$ and $E_{c}=\Delta / 2$. Conductance and voltage are counted in units of $R_{Q} G^{2} /(2 N)$ and $\Delta / e$, respectively.

FIG. 4. The Andreev conductance (Eq. (31), solid line) and its approximation (Eq. (36), dashed line) are plotted as functions of $w=E_{c} / \Delta$ for $0<w<1$. Conductance is counted in units of $R_{Q} G^{2} /(2 N)$. 

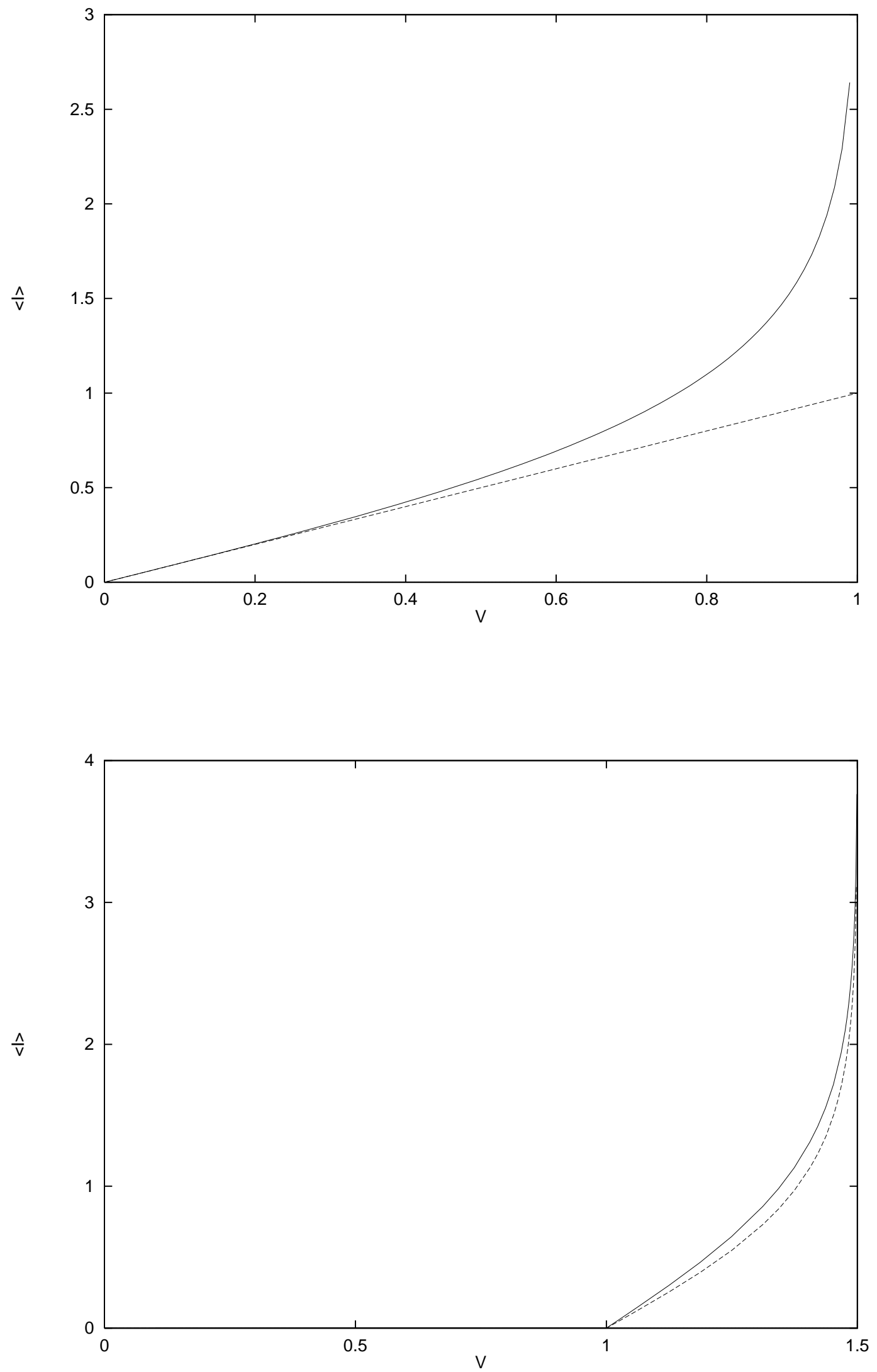

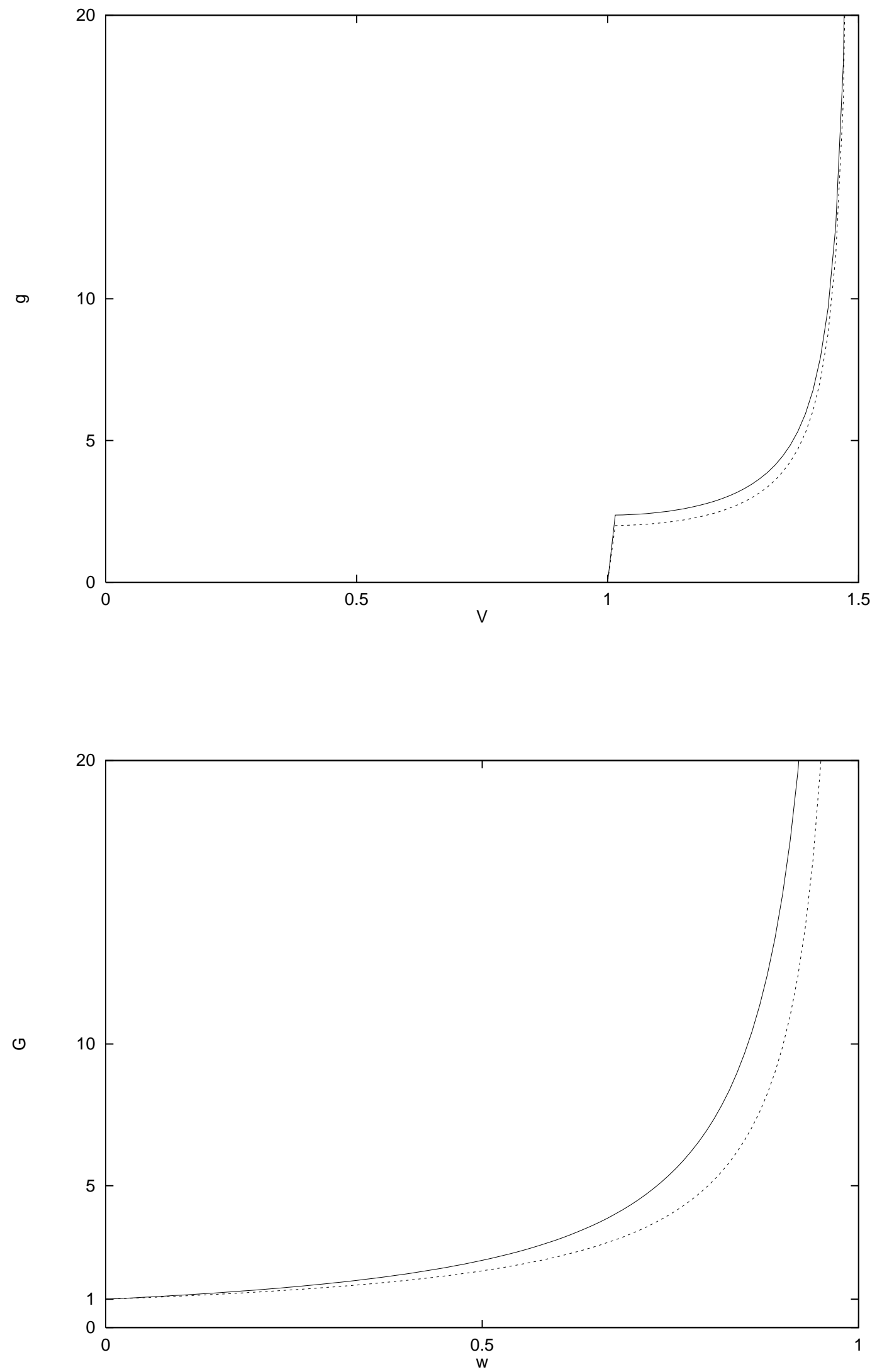